\title{
Re-evaluation of Propagation Rate Coefficient of Free-Radical Polymerization of $N$-Vinylcarbazole by the Rotating-Sector Technique
}

\author{
Masataka OHOKA, ${ }^{\dagger}$ Sadahito Misumi, Hideo OHKITA, Shinzaburo ITO, \\ and Masahide YАMАмОто* \\ Department of Polymer Chemistry, Graduate School of Engineering, Kyoto University, Kyoto 615-8510, Japan \\ ${ }^{*}$ Faculty of Science and Engineering, Ritsumeikan University, Kusatsu 525-8577, Japan
}

(Received June 24, 2004; Accepted September 29, 2004; Published December 15, 2004)

\begin{abstract}
Rotating sector polymerization in conjugation with a size exclusion chromatography (RSP-SEC) was adopted to determine the propagation rate coefficient $\left(k_{\mathrm{p}}\right)$ in free radical solution polymerization of $N$-vinylcarbazole (VCZ). A photoinitiator whose absorption edge is up to $c a .415 \mathrm{~nm}$ was selected to reduce the light excitation of VCZ. The $k_{\mathrm{p}}$ value was obtained by the RSP-SEC method in the low temperature range of $9-30^{\circ} \mathrm{C}$ and was compared with the value obtained previously by the pulse laser polymerization-SEC method (PLP-SEC). The $k_{\mathrm{p}}$ value obtained by the RSP-SEC method in the range of $9-30^{\circ} \mathrm{C}$ were in good agreement with the Arrhenius equation with $A=2.20 \times$ $10^{8} \mathrm{~L} \mathrm{~mol}^{-1} \mathrm{~s}^{-1}, E_{\mathrm{a}}=27.4 \mathrm{~kJ} \mathrm{~mol}^{-1}$ determined previously by the PLP-SEC method in the range of $30-70^{\circ} \mathrm{C}$. The solvent effects on $k_{\mathrm{p}}$ were also studied and the results were discussed in terms of a growing radical-solvent interaction. [DOI 10.1295/polymj.36.953]

KEY WORDS Rotating Sector Method / Size Exclusion Chromatography / Radical Polymerization / Propagation Rate Coefficient / N-Vinylcarbazole / Solvent Effects /
\end{abstract}

The IUPAC working group recommends the pulsed-laser polymerization/size-exclusion chromatography technique (PLP-SEC) as the determination method for the propagation rate coefficients $\left(k_{\mathrm{p}}\right)$ of radical polymerization ${ }^{1}$ and it has been extensively applied to various vinyl monomers. ${ }^{2}$ Previously, we studied the radical polymerization of $N$-vinylcarbazole (VCZ) by the PLP-SEC method, and reported the propagation rate constant with the Arrhenius parameters. ${ }^{3,4}$ Recently Davis' group studied the VCZ system by the PLP method with the $355 \mathrm{~nm}$ excitation and pointed out the complications of this system, such as light excitation of the monomer, some side-reactions besides photopolymerization. ${ }^{5}$ They also reinvestigated the $k_{\mathrm{p}}$ of the same system by the $440 \mathrm{~nm}$ excitation PLP and showed generally good agreement of the experimental data. ${ }^{6}$

As for the method of evaluating the propagation rate coefficients of radical polymerization, Zifferer and Olaj demonstrated theoretically ${ }^{7}$ and experimentally $^{8}$ that the rotating sector polymerization-SEC method (RSP-SEC) gives kinetic parameters that agree quantitatively with those obtained by the PLPSEC method. In RSP we can choose rather freely an excitation wavelength with a moderate intensity for photopolymerization by using a mercury or xenon arc lamp with optical filters.

So far the solvent effects on $k_{\mathrm{p}}$ of radical polymerization have been extensively studied. ${ }^{9-11}$ Experimen- tally the phenomena depend much on the kind of growing radical and solvents. As for styrene that is a typical monomer of electron donating radical, Burnett et al. ${ }^{12}$ reported by using the rotating sector method that the $k_{\mathrm{p}}$ decreased with the increase of benzonitrile concentration and proposed the complex formation of the growing radical with solvents. Kamachi et $a l .{ }^{10}$ studied the solvent effects on $k_{\mathrm{p}}$ for various monomers by the rotating sector method. They found that $k_{\mathrm{p}}$ did not correlate well with Hammett's $\sigma_{\mathrm{p}}$, but it correlated well with the stabilization energy $(\Delta E)$ caused by the interaction of the propagating radical with aromatic solvents. They proposed the solventradical complex formation. ${ }^{13,14}$ Later, some groups reported no evidence for such a solvent effect. ${ }^{15,16}$ However, O'Driscoll et al. ${ }^{17-19}$ studied the $k_{\mathrm{p}}$ of styrene and methyl methacrylate (MMA) by using PLP and showed that the growing styrene radical forms a weak complex with solvents and that MMA radical forms a strong complex with solvents.

Here, we applied the RSP-SEC method to radical polymerization of $N$-vinylcarbazole and studied the kinetic parameters in detail. With the RSP-SEC method we could confirm the results we previously obtained by the PLP-SEC method. ${ }^{3,4}$ We studied the solvent effects on $k_{\mathrm{p}}$ and Arrhenius parameters in several aromatic solvents, and discussed the mechanism of a growing radical interaction with aromatic solvents.

${ }^{\dagger}$ To whom correspondence should be addressed (Fax: 075-383-2617, E-mail: mohoka@ photo.polym.kyoto-u.ac.jp). 


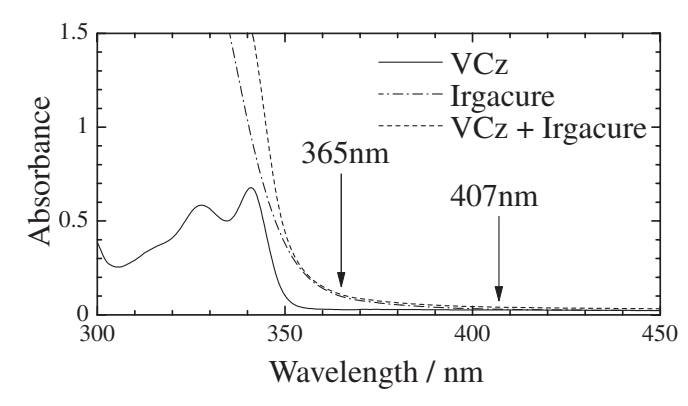

Figure 1. Absorption spectra of $\operatorname{VCZ}\left(0.5 \mathrm{~mol} \mathrm{~L}^{-1}\right)$ and Irgacure $369\left(2 \times 10^{-3} \mathrm{~mol} \mathrm{~L}^{-1}\right)$ and the mixed solution.

\section{EXPERIMENTAL}

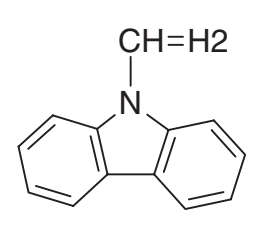

$N$-Vinylcarbazole

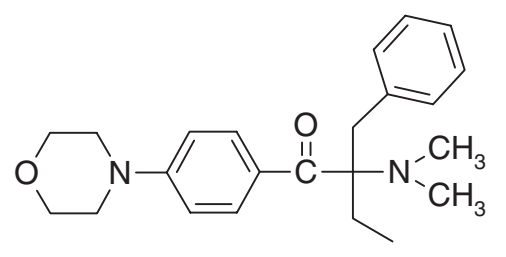

Irgacure369

\section{Procedure}

Materials. An efficient photoinitiator, 2-benzyl-2dimethylamino-1-(4-morpholino-phenyl)-butanone-1 (Irgacure 369, Ciba Geigy) was selected in this study, which has an absorption tail up to $c a$. $415 \mathrm{~nm}$ and absorbs longer wavelength light than VCZ (Figure 1). The quantum yield of $\alpha$-cleavage of this photoinitiator is $0.22 .{ }^{20} \mathrm{VCZ}$ (Nacalai Tesque, extra pure reagent) was purified by recrystallization three times from methanol and hexane. Then, it was dried in vacuo for two days at room temperature. Solvents used here were benzene (Nacalai Tesque, specially prepared reagent), chlorobenzene (Nacalai Tesque, guaranteed reagent), anisole (Nacalai Tesque, guaranteed reagent), benzonitrile (Nacalai Tesque, guaranteed reagent). The specially prepared reagent was used without further purification. Chlorobenzene, anisole, and benzonitrile were purified by distillation under reduced pressure before use.

Equipment. We applied the rotating sector polymerization-SEC method (RSP-SEC) to the free radical solution polymerization of VCZ. In this method, the conventional rotating sector light pulse was used in place of the laser-pulse. The light source was $1 \mathrm{~kW}$ high pressure Hg lamp (Ushio) with a cut-off filter, L-38 (Hoya, transmission: 84\% (407 nm), 10\% $(365 \mathrm{~nm}), 0 \%(347 \mathrm{~nm}))$ and two pieces of heat cutoff filters, the excitation wavelength was mainly $365 \mathrm{~nm}$ and $407 \mathrm{~nm}$. The repetition time of light pulses $\left(t_{0}\right)$ was $227 \mathrm{~ms}$ with the pulse width $\left(t_{\mathrm{w}}\right) 10 \mathrm{~ms}$, giving the fraction of light period $r=0.044$. Molecular weight distribution (MWD) was measured by SEC on an HLC-802UR (Tosoh) system, comprising of a column (Tosoh, TSK-GEL, G4000 HHR $\times 2$ ) and a differential refractometer and UV-detector $(\lambda=340$ $\mathrm{nm})$. The eluent was THF with a flow rate of 0.8 $\mathrm{mL} \mathrm{min}^{-1}$. The MWD analysis was based on universal calibration by using narrow MWD polystyrene standards (Tosoh standard sample) and the MarkHouwink-Sakurada coefficients. This calibration curve was checked with a few samples of narrowMWD poly(vinylcarbazole) (PVCZ) that were prepared by living cationic polymerization and their $M_{\mathrm{w}}$ 's were measured by light scattering (LS).

The concentration of the photoinitiator was adjusted to be $c a \cdot 10^{-2} \mathrm{M}$. The solution in the sample cell $(4 \times 1 \times 1-\mathrm{cm}$ quartz cell) was bubbled with argon gas for $10 \mathrm{~min}$. The irradiation time was for $30-90 \mathrm{~s}$. After the polymerization the solvent was evaporated and dissolved in THF to measure MWD of the polymer.

RSP-SEC Method. The principle of the rotatingsector method is the same as that of PLP. The photoinitiator decomposes during the light pulse and the radical formed initiates the propagation. When the next light pulse arrives, the radical is exposed to a high concentration of newly generated radicals, which lead to their termination with a greatly increased probability. If a propagating radical is terminated by the $i$-th pulse after the initiating pulse, the chain length $L_{i}$ of the polymer formed is determined by the following equation:

$$
L_{i}=i k_{\mathrm{p}}[M] t_{0}
$$

where $[M]$ is the monomer concentration, $t_{0}$ is the repetition time between pulses and $i$ is an integer.

Zifferer and Olaj ${ }^{7}$ showed theoretically the condition at which eq 1 is valid when the fraction of light period $r\left(=t_{\mathrm{w}} / t_{0}\right)$ and $t_{0} / \tau_{\mathrm{s}}$ are varied, where $t_{0}, t_{\mathrm{w}}$, $\tau_{\mathrm{s}}$ are the repetition time between pulses, the pulse width, the mean lifetime of a radical under the stationary illumination, respectively. In the case of $r=0.044$ that was adopted in this study, the ratio $t_{0} / \tau_{\mathrm{s}}$ should be less than 15 and this condition was fulfilled in our experimental setup: $t_{0} / \tau_{\mathrm{s}}<10$.

MO Calculation. To check the possibility of the complex formation of the propagating radical with solvent molecules, we calculated the delocalization stabilization $(\Delta E)$ for the complex formation according to Kamachi's model (ref 10, Model 1 in Figure 4). The parameters for simple LCAO MO calculation were adopted from ref 21 . The delocalization stabilization $(\Delta E)$ was calculated by the following formula. $^{21}$ 


$$
\begin{aligned}
-\Delta E= & \frac{2\left(\sum_{i}^{\text {occ unocc }} \sum_{j}^{\text {occ-N unocc }}-\sum_{j} \sum_{i}\right)\left(d_{n}^{i}\right)^{2}\left(C_{r}^{j}+C_{s}^{j}\right)^{2}(\gamma \beta)^{2}}{\left(E_{i}-E_{j}\right)} \\
+ & \frac{\left(\sum_{j}^{\text {occ }}-\sum_{j}^{\text {unocc }}\right)\left(d_{n}^{N}\right)^{2}\left(C_{r}^{j}+C_{s}^{j}\right)^{2}(\gamma \beta)^{2}}{\left(E_{N}-E_{j}\right)}
\end{aligned}
$$

where $E_{N}$ and $E_{i}$ are the energies of the nonbonding $\mathrm{MO}$ and the occupied (unoccupied) MO of the propagating radical, respectively, and $E_{j}$ is the energy of occupied (unoccupied) MO of the solvent. The value $\gamma \beta$ is the resonance integral between the radical and the $r$ th and sth atoms of the aromatic solvent. Notation $C_{r}^{j}$ and $C_{s}^{j}$ are the coefficients of the $r$ th and sth carbon AO's of the solvent in which the $r$ th and $s$ th carbon atoms are adjacent to each other. Notation $d_{n}^{N}$ and $d_{n}^{i}$ are the coefficients of the carbon AO of the propagating radical in the nonbonding $\mathrm{MO}$ and the occupied (unoccupied) MO, respectively.

\section{RESULTS AND DISSCUSION}

\section{The $k_{p}$ Obtained by RSP-SEC Method}

Figure 2 shows the molecular weight distribution of the polymer formed by irradiation at $30^{\circ} \mathrm{C}$. We could observe at least two peaks, $L_{1}$ and $L_{2}$ in the first derivative $\mathrm{d} w(\log M) / \mathrm{d}(\log M)$. Here, the relationship between the first peak and the second peak: $L_{2}=$ $2 \times L_{1}$ was satisfied. Figure 3 shows the relation between $L_{1}$ and the monomer concentration. As the monomer concentration increased, the inflection point $L_{1}$ shifted linearly toward a higher side: the result shows the validity of eq 1 . Then we calculated the propagation rate coefficient $k_{\mathrm{p}}$ from $L_{1}$ by using eq 1. Figure 4 shows the Arrhenius plot of the propagation rate coefficients. The relation between $\ln k_{\mathrm{p}}$ and

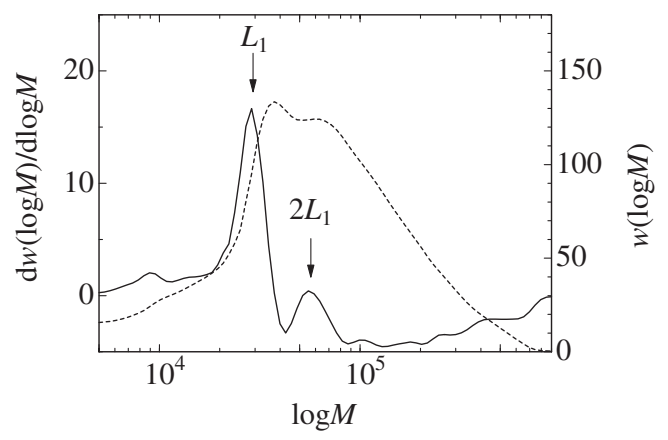

Figure 2. Molecular weight distribution $w(\log M)$ (solid line) and the first derivative $\mathrm{d} w(\log M) / \mathrm{d}(\log M)$ (broken line) of the polymer formed in RSP experiment for VCZ. VCZ: $1 \mathrm{~mol} \mathrm{~L}^{-1}$; Irgacure 369: $2 \times 10^{-3} \mathrm{~mol} \mathrm{~L}^{-1}$; solvent: benzene: temperature: $30^{\circ} \mathrm{C}$. The inflection points are shown as $L_{i}$.

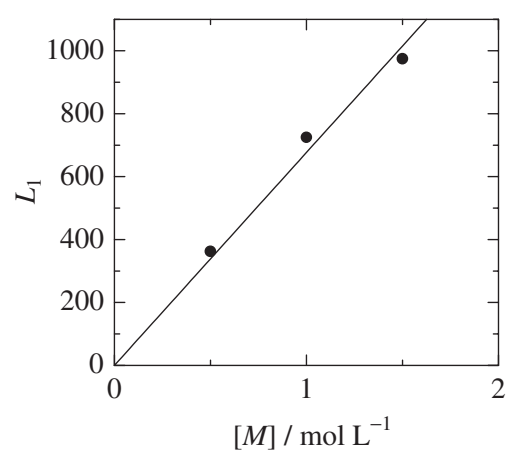

Figure 3. Effect of monomer concentration on $L_{1}$. Irgacure 369: $2 \times 10^{-3} \mathrm{~mol} \mathrm{~L}^{-1}$; solvent: benzene; temperature: $30^{\circ} \mathrm{C}$; RSP: $t_{0}=227 \mathrm{~ms}, t_{\mathrm{w}}=10 \mathrm{~ms}$.

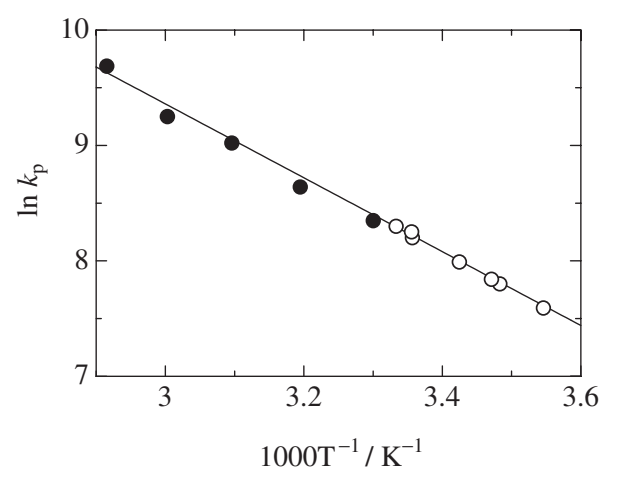

Figure 4. Arrhenius plot for the rate coefficient $k_{\mathrm{p}}$ determined by the present RSP $(\bigcirc)$ and the previous PLP (

$1 / T$ obtained by the RSP-SEC method (solid circle, from 9 to $30^{\circ} \mathrm{C}$ ) is shown in addition to those obtained by the PLP-SEC method (circle, from 30 to $70^{\circ} \mathrm{C}$ ) reported previously. ${ }^{4}$ The values obtained by the RSPSEC method fitted the Arrhenius equation well with $A=2.20 \times 10^{8} \mathrm{~L} \mathrm{~mol}^{-1} \mathrm{~s}^{-1}$ and $E_{\mathrm{a}}=27.4 \mathrm{~kJ} \mathrm{~mol}^{-1}$ that was obtained by PLP-SEC method previously. This means that the Arrhenius equation holds in a rather wide temperature range of $9-70^{\circ} \mathrm{C}$, and furthermore confirms our previous findings.

\section{Solvent Effects}

Figure 5 shows Arrhenius plots of $k_{\mathrm{p}}$ in several aromatic solvents. Table I gives the $k_{\mathrm{p}}$ at $25^{\circ} \mathrm{C}$ and Arrhenius parameters with various solvent parameters as the reference. The variation of $k_{\mathrm{p}}$ values is within a 2fold range, depending on the kind of solvents used in this study: the $k_{\mathrm{p}}$ value in benzonitrile solvent is a half of that in benzene solvent.

The solvent effects on $k_{\mathrm{p}}$ can be discussed in terms of the following points: (1) viscosity of the medium, (2) polarity of the solvents, (3) the growing radicalsolvent complex formation. Table I shows that the variation of $k_{\mathrm{p}}$ with different solvents is within a 2fold range and can be discussed with the dynamic 
Table I. The $k_{\mathrm{p}}$ with Arrhenius parameters $\left(A, E_{\mathrm{a}}\right)$; viscosity $(\eta)$, dielectric constant $(\varepsilon)$, Hammett's $\sigma_{\mathrm{p}}$ of aromatic solvents; stabilization energy $(\Delta E)$ of the growing radical of $N$-vinylcarbazole

\begin{tabular}{lccccccc}
\hline \multicolumn{1}{c}{ Solvent } & $\begin{array}{c}k_{\mathrm{p}} \text { at } 25^{\circ} \mathrm{C} \\
\left(\times 10^{3} \mathrm{~L} \mathrm{~mol}^{-1}\right)\end{array}$ & $\begin{array}{c}A \\
\left(\times 10^{8} \mathrm{~L} \mathrm{~mol}^{-1} \mathrm{~s}^{-1}\right)\end{array}$ & $\begin{array}{c}E_{\mathrm{a}} \\
\left(\mathrm{kJ} \mathrm{mol}^{-1}\right)\end{array}$ & $\begin{array}{c}\eta \\
\left(\mathrm{cp}, 25{ }^{\circ} \mathrm{C}\right)\end{array}$ & $\begin{array}{c}\varepsilon \\
\left(25{ }^{\circ} \mathrm{C}\right)\end{array}$ & $\begin{array}{c}\sigma_{\mathrm{p}} \\
\left(-\gamma^{2} \beta\right)\end{array}$ \\
\hline Benzene & $3.47 \pm 0.14$ & 2.20 & 27.4 & 0.61 & 2.3 & 0 & 0.272 \\
Chlorobenzene & $2.49 \pm 0.13$ & 1.58 & 27.4 & 0.75 & 5.6 & 0.23 & 0.525 \\
Anisole & $2.38 \pm 0.09$ & 1.51 & 27.4 & 1.01 & 4.3 & -0.27 & 0.745 \\
Benzonitrile & $1.73 \pm 0.14$ & 0.29 & 24.1 & 1.24 & 25.2 & 0.66 & 0.800 \\
THF & $1.91 \pm 0.15$ & - & - & 0.46 & 7.6 & - & - \\
\hline
\end{tabular}

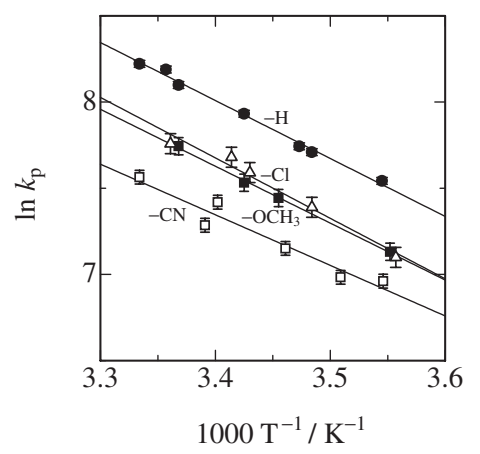

Figure 5. Arrhenius plots for $k_{\mathrm{p}}$ in various solvents. Benzene $(\bullet)$, chlorobenzene $(\triangle)$, anisole $(\square)$, benzonitrile $(\square)$.

theory of viscosity effects, ${ }^{11}$ but the $k_{\mathrm{p}}$ values do not correlate well with viscosity of solvents.

As for the polarity effect of solvents, dipolar reactants and the transition state of the reaction may be effected by the polarity of solvent $(\varepsilon)$, where $\ln k_{\mathrm{p}}$ should vary with $1 / \varepsilon$ or $(\varepsilon-1) /(2 \varepsilon+1) .{ }^{22}$ Figure 6 shows a good relationship between $\ln k_{\mathrm{p}}$ and $1 / \varepsilon$ for all the solvents examined in this study: the $k_{\mathrm{p}}$ decreased with the increase of solvent polarity, i.e., there is a 2 -fold deceleration in going from benzene to benzonitrile. This means that in polar solvents, the dipolar reactants are more stabilized than less dipolar activated complex. In other words, a growing radical is weakly solvated by polar solvents. This mechanism is similar to the radical-solvent complex model discussed in the next section.

As for the radical-solvent complex mechanism,

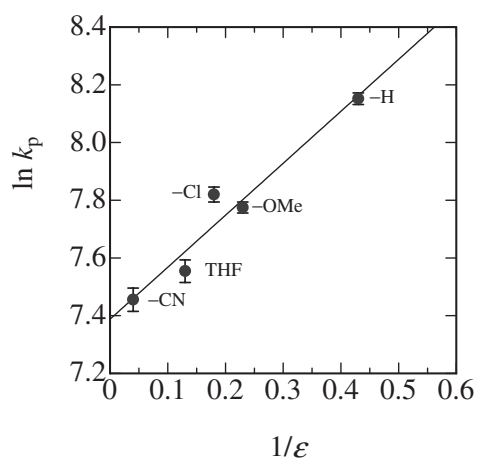

Figure 6. Relation between $k_{\mathrm{p}}$ and $1 / \varepsilon$.
Kamachi et al. ${ }^{13}$ proposed the radical-solvent complex formation in aromatic solvents for various monomers. They demonstrated that the $k_{\mathrm{p}}$ values do not have a good correlation with the Hammett's $\sigma_{\mathrm{p}}$, but rather have a good correlation with the stabilization energy $(\Delta E)$ that is caused by the interaction of the propagating radical with aromatic solvents. From these results they proposed the radical-solvent complex formation. Similar results were obtained in this study. The $k_{\mathrm{p}}$ values did not correlate well with Hammett's $\sigma_{\mathrm{p}}$ (Table I), but they have a rather good correlation with the stabilization energy $(\Delta E)$ of the radical with aromatic solvents (Figure 7$)$. The $(Q, e)$ value of VCZ monomer for radical polymerization is $(0.41,-1.40)$, the $e$ value of which means the $\mathrm{VCZ}$ radical is of electron-rich nature. These results may suggest that the electron-rich growing radical interacts with an electron acceptor solvent to form a weak complex.

According to the radical-solvent complex model, ${ }^{10}$ the propagating radical forms a reversible complex with a solvent, resulting in a dormant state, then the active growing radical concentration is reduced and the apparent $k_{\mathrm{p}}$ is lowered. If this is the case, apparent activation energy $E_{\mathrm{a}}$ will be almost the same and the pre-exponential factor $A$ will decrease with increasing stabilized growing radical. The variation of $A$ and nearly constancy of $E_{\mathrm{a}}$ observed experimentally (Table I) may suggest the formation of weak complex of the growing radical end with aromatic solvents. The solvation of the growing radical by polar solvents that was led by the polarity effect in the previous sec-

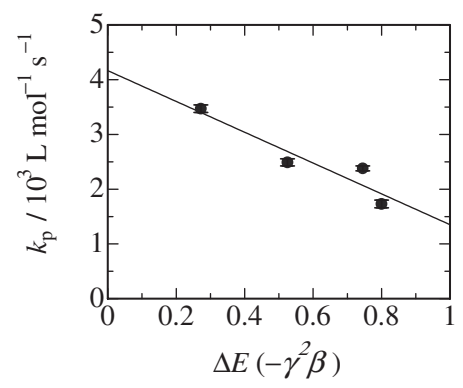

Figure 7. Relation between $k_{\mathrm{p}}$ and the delocalization stabilization energy $\Delta E$. 
tion gives a similar physical picture. However, no direct evidence for the formation of the radical-solvent complex or the solvation of the growing radical by polar solvents has been given yet. Further study with spectroscopic methods such as ESR, laser photolysis is needed to prove a radical-solvent interaction model and this is in progress.

\section{The $k_{p}$ in a Wide Temperature Range}

The results obtained in this study cleared up the complications pointed out by Davis' group. ${ }^{5}$ One is the excitation light wavelength we selected to excite the photoinitiator dominantly and to reduce the excitation of VCZ monomer. Actually no photopolymerization was observed without the photoinitiator and the whole fraction of the obtained polymer was easily soluble in various solvents. This means that there is no side reaction product such as crosslinked polymer. The excitation light intensity was also much weaker than that of the laser intensity, but the efficiency of radical production for the photoinitiator is very high: the quantum yield of $\alpha$-cleavage is 0.22 . This is one reason we could apply the RSP-SEC method in the present study.

Figure 8 shows the Arrhenius plots obtained in the present study, and those reported by Davies' group with PLP-SEC $\left(355 \mathrm{~nm}, 20 \text { to }-20^{\circ} \mathrm{C}\right)^{5}$ and with PLP-SEC $\left(440 \mathrm{~nm}, 0-50{ }^{\circ} \mathrm{C}\right){ }^{6}$ There is some deviation between the previous line and that of Davies' group: the slope in this study is slightly higher than that by Davies' group. However, the temperature range of our data and theirs is different, i.e., our data covers a rather high temperature range and theirs a low temperature range. When the experimental condition is scrutinized in detail, the Arrhenius plot is not necessarily linear, i.e., the temperature dependence of viscosity and dielectric constants of solutions may not change linearly with a wide temperature range. The $A$ and $E_{\mathrm{a}}$ values are different in each study, but

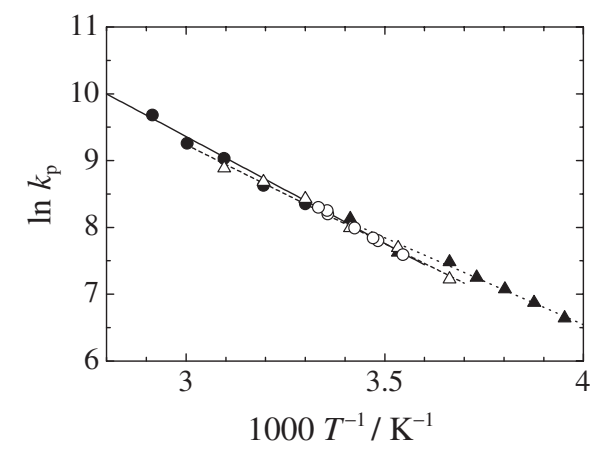

Figure 8. Arrhenius plots of $k_{\mathrm{p}}$ determined by the present RSP-SEC $(\bigcirc)$ and the previous PLP-SEC $(\bullet)$ by our group; $\operatorname{PLP}(355 \mathrm{~nm})-\operatorname{SEC}(\boldsymbol{\Delta})$ and PLP $(440 \mathrm{~nm})-\operatorname{SEC}(\triangle)$ by Davis' group. the agreement of each experimental data is rather good. We are further studying this point.

\section{CONCLUSIONS}

The present results indicate the applicability of the RSP-SEC method for evaluation of the propagation rate coefficient of free radical polymerization. In the RSP-SEC method, the photon density is much lower than the laser pulse and visible light excitation of the photoinitiator may exclude the light excitation of the VCZ monomer. Further the polymerization proceeded at rather lower temperatures. The results obtained in this study are in good agreement with the previous one obtained by the PLP-SEC method. Solvent effects on $k_{\mathrm{p}}$ and Arrhenius parameters were analyzed and the result was explained by the mechanism of solvent-radical complex formation or the solvation of a growing radical with solvents.

\section{REFERENCES}

1. M. Buback, R. G. Gilbert, R. A. Hutchinson, B. Klumperman, F. Kuchta, and B. G. Manders, Macromol. Chem. Phys., 196, 3267 (1995).

2. R. A. Hutchinson, J. R. Richards, and M. T. Aronson, Macromolecules, 27, 4530 (1994).

3. M. Ohoka, S. Misumi, and M. Yamamoto, Polym. J., 31, 316 (1999).

4. M. Ohoka, S. Misumi, and M. Yamamoto, Polym. J., 31, 878 (1999).

5. M. Yin, C. Barner-Kowollik, J. P. A. Heuts, and T. P. Davis, Macromol. Rapid Commun., 22, 1035 (2001).

6. M. Yin, T. P. Davis, J. P. A. Heuts, and C. Barner-Kowollik, Macromol. Rapid Commun., 24, 408 (2003).

7. G. Zifferer and O. F. Olaj, Makromol. Chem., 191, 1699 (1990).

8. O. F. Olaj, P. Kremminger, and I. Schnöll-Bitai, Makromol. Chem., Rapid Commun., 9, 771 (1988).

9. G. Moad and D. H. Solomon, "The Chemistry of Free Radical Polymerization,” Pergamon, Oxford, U.K., 1995, chapt. 7.

10. M. Kamachi, Adv. Polym. Sci., 38, 56 (1981).

11. B. R. Morrison, M. C. Piton, M. A. Winnik, R. G. Gilbert, and D. H. Napper, Macromolecules, 26, 4368 (1993).

12. G. M. Burnett, G. G. Cameron, and S. N. Joiner, J. Chem. Soc., Faraday Trans. I, 69, 322 (1973).

13. M. Kamachi, D. J. Liaw, and S. Nozakura, Polym. J., 11, 921 (1979).

14. M. Kamachi, D. J. Liaw, and S. Nozakura, Polym. J., 13, 41 (1981).

15. T. P. Davis, K. F. O'Driscoll, M. C. Piton, and M. A. Winnik, Macromolecules, 22, 2785 (1989).

16. S. Beuermann, M. Buback, and G. T. Russell, Macromol. Rapid Commun., 15, 647 (1994).

17. K. F. O'Driscoll, M. J. Monteiro, and B. M. Klumperman, J. Polym. Sci., Part A: Polym. Chem., 35, 515 (1997). 
18. K. F. O'Driscoll, T. P. Davis, B. M. Klumperman, and E. L. Madruga, Macromol. Rapid Commun., 16, 207 (1995).

19. M. D. Zammit, T. P. Davis, G. D. Willett, and K. F. O'Driscoll, J. Polym. Sci., Part A: Polym. Chem., 35, 2311 (1997).

20. V. Desobry, K. Dietliker, R. Hüsler, L. Misev, M. Rembold, G. Rist, and W. Rutsch, in "Radiation Curing of Polymeric Materials," C. E. Hoyle and J. F. Kinstle, Ed., ACS Sympo- sium Series No. 417, American Chemical Society, Washington, D.C., 1990, p 92.

21. T. Yonezawa, T. Nagata, H. Kato, A. Imamura, and K. Morokuma, "Introduction to Quantum Chemistry, 3rd ed.," Vol. 1, Kagakudojin, Kyoto 1986.

22. C. Reichardt, "Solvents and Solvent Effects in Organic Chemistry, 2nd ed.," VCH, Weinheim 1988, chapt. 5. 